\title{
EVALUACIÓN DE PTERDOFITAS EN BOSQUES DE LA ZONA RESERVADA ALLPAHUAYO - MISHANA Y SANTA ROSA, LORETO*
}

\author{
Glenda Cárdenas ${ }^{1}$, Carolina Aramburu ${ }^{2}$, Lidia Castro ${ }^{3}$, Alberto García ${ }^{3}$, Adriana Zegarra ${ }^{4}$ y Manuel \\ Flores $^{3}$
}

\begin{abstract}
RESUMEN
El presente estudio fue realizado en la Zona Reserva Allpahuayo - Mishana y en el poblado de Santa Rosa (km 26 y 60 respectivamente, de la carretera Iquitos-Nauta), con la finalidad de evaluar la población de pteridofitas en distintos tipos de bosque en el marco del curso "Uso de inventarios biológicos para revelar patrones de distribución geográfica de especies en la selva baja amazónica" organizado por el proyecto Diversidad Biológica de la Amazonía Peruana, Perú - Finlandia (BIODAMAZ), con el apoyo del Instituto de Investigaciones de la Amazonía Peruana (IIAP) y la Universidad Nacional de la Amazonía Peruana (UNAP). Fueron realizados inventarios en siete transectos de $200 \times 5 \mathrm{~m}$, divididos en ocho subunidades de $25 \times 5 \mathrm{~m}$. Las zonas de muestreo fueron seleccionadas subjetivamente para representar geológicamente distintas áreas. Se colectó pteridofitas hasta una altura de $2 \mathrm{~m}$. Fueron establecidas relaciones entre la composición de especies en los transectos y la cantidad de materia orgánica por transecto. Las ordenaciones fueron visualizadas con la prueba de Mantel. Existe una correlación entre los cambios de grosor de materia orgánica y los cambios florísticos entre los transectos, pero con alto riesgo de error $(\mathrm{r}=0.28, \mathrm{p}=0.11)$. En el caso del varillal húmedo, la correlación fue negativa $(0.25)$, indicando que en este transecto no existe una relación entre materia orgánica y composición de especies de helechos.
\end{abstract}

Palabras clave: Allpahuayo - Mishana, inventario, Loreto, materia orgánica, pteridofitas, Santa Rosa.

\begin{abstract}
This study was conducted in the Allpahuayo-Mishana Reserved Zone and in the village of Santa Rosa (km 26 and 60 on the Iquitos-Nauta road, respectively) with the aim of evaluating the Pteridophyta population in different types of forests as part of the "Use of biological inventories to reveal geographic distribution patterns of lowland Amazonian species" course organized by the Peru-Finland Biological Diversity of the Peruvian Amazon project (BIODAMAZ), with the support of the Peruvian Amazonian Research Institute (IIAP) and the Peruvian Amazonian National University (UNAP). Inventories were made of seven transects of $200 \times 5 \mathrm{~m}$, divided into eight subunits of $25 \times 5 \mathrm{~m}$. The sampling areas were selected subjectively in order to include various geologically different areas. Ferns of up to $2 \mathrm{~m}$ high were collected. The relationships between the species composition in the transects and the quantity of organic material in the transects were studied. The ordinations were visualized using the Mantel test. There is correlation between the thickness of the organic material layer and floristic changes in the transects, but with a high risk of error $(r=0.28, p=0.11)$. In the case of the humid white-sand forest, the correlation was negative $(0.25)$ indicating that in this transect there was no relation between the organic material and fern species composition.
\end{abstract}

Key words: Allpahuayo-Mishana, inventory, Loreto, organic material, Pteridophyta, Santa Rosa.

* Referencia bibliográfica del artículo: Cárdenas, G., Aramburu, C., Castro, L., García, A., Zegarra, A. \& Flores, M. 2002. Evaluación de pteridofitas en bosques de la Zona Reservada Allpahuayo - Mishana y Santa Rosa, Loreto. En: Rodríguez, J.J., Ruokolainen, K. \& Soini, P. (eds.). La diversidad biológica de la Zona Reservada Allpahuayo - Mishana, Loreto, Perú: Relaciones entre distribución, abundancia y hábitats. Folia Amazónica 14 (1-2): NÚMEROS DE PÁGINA.

1 Universidad Nacional de la Amazonía Peruana (UNAP), Iquitos, Perú; correo electrónico: biodamaz@iiap.org.pe

2 Museo de Historia Natural, Universidad Nacional Mayor de San Marcos (UNMSM), Lima, Perú.

3 Universidad Nacional de la Amazonía Peruana (UNAP), Iquitos, Perú.

4 Universidad Nacional San Antonio Abad del Cusco (UNSAAC), Cusco, Perú. 


\section{INTRODUCCIÓN}

Los bosques de la Amazonía baja cercanos a la ciudad de Iquitos se caracterizan por poseer gran riqueza florística, debido a la presencia de mosaicos edáficos y topográficos, lo que influencia la distribución y abundancia de las especies. Es importante conocer la distribución de especies de plantas y tratar de entender las distribuciones con relación a su ambiente. Se sabe que un sitio específico con características especiales alberga a aquellas especies que están adaptadas a dichas condiciones ambientales, las cuales muchas veces sirven como "indicadoras" de determinados hábitats.

Es conocido que las especies de pteridofitas se encuentran en diferentes tipos de suelos (Tuomisto y Ruokolainen, 1994; Tuomisto y Poulsen, 1996), están representadas en todos los tipos de bosque que se conocen en el área, su situación taxonómica es mejor entendida que con la mayoría de especies amazónicas, y tienen pocas especies utilizadas por la gente, por lo tanto podrían servir como indicadoras de ciertas condiciones edáficas (Tuomisto y Ruokolainen, 1998).

El objetivo del estudio fue contribuir al conocimiento de las características ambientales que afectan la distribución de helechos en la selva amazónica. Específicamente, se trató de ver si la composición de especies de helechos varía según el grosor de la capa de materia orgánica superficial del suelo. Este trabajo se realizón en el marco del curso "Uso de inventarios biológicos para revelar patrones de distribución geográfica de especies en la selva baja amazónica" organizado por el proyecto Diversidad Biológica de la Amazonía Peruana, Perú - Finlandia (BIODAMAZ) $^{5}$, con el apoyo del Instituto de Investigaciones de la Amazonía Peruana (IIAP) y la Universidad Nacional de la Amazonía Peruana (UNAP).

\section{MATERIAL Y MÉTODO}

\section{1. Áreas de muestreo}

Las zonas de muestreo del presente trabajo estuvieron ubicadas en la Zona Reservada Allpahuayo - Mishana y en el poblado de Santa Rosa, a la altura del km 26 y 60 respectivamente de la carretera Iquitos - Nauta.

Las zonas de muestreo fueron seleccionadas subjetivamente, ya que fueron zonas de fácil acceso, muchas de ellas conocidas e intervenidas en investigaciones anteriores.

El método utilizado para la toma de datos fue el transecto en faja, siguiendo 7 trochas de $200 \mathrm{~m}$ de largo y $5 \mathrm{~m}$ de ancho, divididas en 8 subunidades de $25 \mathrm{~m}$ x $5 \mathrm{~m}$. Los transectos fueron elegidos tratando de abarcar geológica y geomorfológicamente diferentes tipos de bosque, como son: bosques en suelos de la Formación Pebas, varillal seco, bosque de terraza, bosque de quebrada inundable en Formación Pebas (transectos "Pebas" y "Medicinales"), bosque en Formación Pebas tardío (Santa Rosa), bosque ocasionalmente inundado en Formación Pebas ("Supaychacra"), bosque en suelos arcilloso-arenosos de terraza de quebrada ("Terraza"), y bosque en suelos de arena blanca (Varillal Seco y Varillal Húmedo).

Se registró el número de individuos por especie y se colectó muestras representativas de cada especie terrestre y/o epífita, desde el suelo hasta una altura de $2 \mathrm{~m}$. Las plantas colectadas fueron trasladadas al Herbarium Amazonense (AMAZ) de la UNAP, donde se realizó el proceso de herborización e identificación, con ayuda de las claves taxonómicas (Tryon y Stolze, 1989a; 1989b; 1991; 1992; 1993; 1994), y colecciones del Herbario.

En cada subunidad de muestreo se registró el grosor de la capa de materia orgánica del suelo en el centro de la subunidad (Figura 1).

5 Proyecto Diversidad Biológica de la Amazonía Peruana (BIODAMAZ) es un convenio entre los gobiernos del Perú y de Finlandia ejecutado conjuntamente por el Instituto de Investigaciones de la Amazonía Peruana (IIAP) y por el consorcio finlandés formado por la empresa de consultorías ambientales, Biota BD Oy, y por la Universidad de Turku. 


\subsection{Análisis estadístico}

Para comparar las unidades de muestreo, se aplicó el índice de similitud de Steinhaus, en el caso de composición de especies, y la distancia euclidiana en el caso de materia orgánica. Antes de los análisis, se convirtió los valores del índice de Steinhaus a su complemento para conseguir dos matrices de distancia.

-Se estudió la correlación entre la composición de especies y el grosor de la capa de materia orgánica, tanto entre los transectos como dentro del transecto de Varillal Húmedo. Se eligió este transecto para hacer la prueba, por ser el transecto con mayor diferencia en el grosor de la capa de materia orgánica entre subunidades. En ambos casos, se estimó la probabilidad de error en la correlación entre matrices con base en 1000 aleatorizaciones.

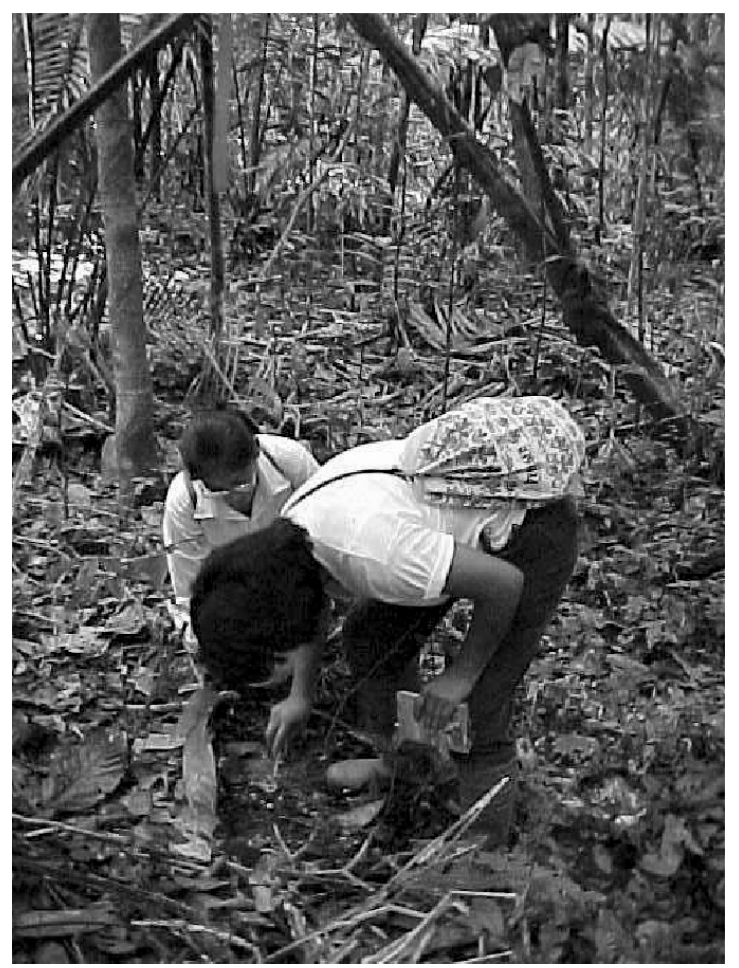

Figura 1. Parte del equipo de investigadores mide la profundidad de la capa de materia orgánica para establecer relaciones entre ésta y la composición de helechos en determinado hábitat. La Zona Reservada Allpahuayo - Mishana presenta una gran diversidad de hábitats. Foto: @IIAP-BIODAMAZ/J.J. Rodriguez

\section{RESULTADOS}

En el presente estudio se registró un total de 71 especies y 11237 individuos de pteridofitas, distribuidas en seis transectos de la Zona Reservada Allpahuayo - Mishana, y un transecto en la zona de Santa Rosa.

El número de especies varió entre 6 y 30, y el número de individuos entre 116 y 5170 en los transectos (Tabla 1).

El mayor número de especies fue encontrado en el transecto Supaychacra, con 30 especies, mientras que el mayor número de individuos (5 170) fue hallado en el transecto Varillal Húmedo.

Claramente existe una correlación positiva entre el número de individuos de helechos y el grosor de la capa de materia orgánica en los transectos. Sin embargo, el número de especies no está correlacionado con ninguna de estas dos variables. 
Tabla 1. Número de especies, individuos y el grosor de la capa de materia orgánica por transecto.

\begin{tabular}{|l|r|r|r|r|r|r|r|}
\cline { 2 - 8 } \multicolumn{1}{c|}{} & Varillal Húmedo & Itaya & Medicinales & Pebas & Varillal Seco & Terraza & Supaychacra \\
\hline $\mathrm{N}^{\circ}$ Especies & 9 & 11 & 25 & 18 & 6 & 20 & 30 \\
$\mathrm{~N}^{\circ}$ Individuos & 5170 & 2702 & 799 & 933 & 116 & 208 & 1309 \\
Prom. materia orgánica (cm) & 17.4 & 3 & 2.4 & 1.2 & 0.5 & 6 & 1 \\
\hline
\end{tabular}

ntre los transectos hubo una correlación de $0.28(\mathrm{p}=0.115)$ entre la composición de especies y el grosor de la capa de materia orgánica. Dentro del transecto de Varillal Húmedo hubo una correlación de -0.25 entre la composición de especies y el grosor de materia orgánica.

En lo que se refiere al índice de similitud de Steinhaus para comparar los siete transectos de acuerdo a la composición de especies, se encontró que existe una similitud mediana entre los transectos de Terraza y Varillal Seco, con un valor de 0.41358 , los cuales comparten dos especies de un total de 20 y 6 especies respectivamente, siendo éstas Lindsaea divaricata Klotzsch y Cyclodium meniscioides (Willd.) C. Presl.

Se ha podido encontrar que algunas especies de helechos son más abundantes en ciertas características del suelo. Lindsaea divaricata es más abundante en zonas poco húmedas de suelo arenoso; Trichomanes martiusii C. Presl, en suelo arenoso, mientras que Adiantum humile Kunze abunda en zonas húmedas y suelos arcillosos.

Por otro lado, en el transecto de Varillal Húmedo existen solamente 9 especies y, sin embargo, el número de individuos es elevado (5 170), abundancia que parece asociada con una capa gruesa de materia orgánica en el suelo (17.4cm). La especie Trichomanes martiusii fue la más abundante, con 3776 individuos.

\section{DISCUSIÓN}

Los resultados de la prueba de Mantel indican que los cambios en la composición florística de las pteridofitas entre los transectos no guardan alta relación con la variación en el grosor de la materia orgánica; además, la correlación observada tiene una probabilidad relativamente alta de error. Tampoco existe una correlación entre el número de especies y el grosor de la capa de materia orgánica. Sin embargo, la cantidad de individuos de helechos está positivamente correlacionada con el grosor de la capa de materia orgánica.

Dentro del transecto que tuvo mayor variación en el grosor de la capa de materia orgánica, no se pudo observar una correlación entre los patrones de semejanzas florísticas y las semejanzas en el grosor de la capa de materia orgánica. La correlación negativa observada no tiene un sentido ecológico, porque implicaría que las zonas más semejantes se dan en términos del grosor de materia orgánica.

Estas observaciones se pueden interpretar como que posiblemente (con un grado relativamente alto de error), entre los tipos diferentes de bosque, hay diferencias en el grosor de materia orgánica que influyen en la composición de especies de helechos. Sin embargo, dentro de un tipo de bosque la variación en la materia orgánica ya no es significativa aparentemente para la composición de especies.

En cuanto a la similitud entre transectos, se observó que hay una semejanza mediana en cuanto a la composición de especies, ya que varios transectos comparten algunas de las especies.

\section{CONCLUSIONES}

El grosor de la capa de materia orgánica del suelo no fue un factor determinante de la diversidad de especies de helechos, pero sí de la abundancia de individuos dentro de los siete transectos. 
Se sugiere que algunas especies de helechos son especialistas de microambientes de textura de suelo y humedad. Estas especies son Lindsaea divaricata, Trichomannes martiusii, y Adiantum humile.

\section{AGRADECIMIENTOS}

Nuestro agradecimiento al proyecto BIODAMAZ, Perú - Finlandia por la oportunidad de realizar este estudio. A la Universidad Nacional de la Amazonía Peruana por el préstamo de las instalaciones del Herbarium Amazonense. A Kalle Ruokolainen por sus valiosas enseñanzas y ayuda en el procesamiento de información y análisis estadístico. A Manuel Flores por su colaboración en la identificación de las muestras botánicas.

\section{BIBLIOGRAFÍA}

TRYON, R.; STOLZE, G. 1989a. Pteridophyta of Peru. Parte I. 1. Ophioglossaaceae-12. Cyatheaceae. Fieldiana: Botany, ns. \# 20.

TRYON, R.; STOLZE, G. 1989b. Pteridophyta of Peru. Parte II. 13. Pteridaceae-15. Dennstaedtiaceae. Fieldiana: Botany, ns. \# 22.

TRYON, R.; STOLZE, G. 1991. Pteridophyta of Peru. Parte IV. 17. Dryopteridacea. Fieldiana: Botany, ns. \# 27.

TRYON, R.; STOLZE, G. 1992. Pteridophyta of Peru. Parte III. 16. Thelypteridaceae. Fieldiana: Botany, ns. \# 29.

TRYON, R.; STOLZE, G. 1993. Pteridophyta of Peru. Parte V. 18. Aspleniaceae-21. Polipodiaceae. Fieldiana: Botany, ns. \# 32.

TRYON, R.; STOLZE, G. 1994. Pteridophyta of Peru. Parte VI. 22. Marsileaceae-28. Isoetaceae. Fieldiana: Botany, ns. \# 34.

TUOMISTO, H.; POULSEN, A. 1996. Influence of edaphic specialization on pteridophyte distribution in Neotropical rain forests. Journal Biogeography 23: 283-293.

TUOMISTO, H.; RUOKOLAINEN, K. 1994. Distribution of Pteridophyta and Melastomataceae along an edaphic gradient in an Amazonian rain forest. J. Veget. Sci. 5 (1): 25-34.

TUOMISTO, H.; RUOKOLAINEN, K. 1998. Uso de especies indicadoras para determinar características del bosque y de la tierra. En: Kalliola, R.; Flores Paitán, S. (eds.). Geoecología y desarrollo amazónico: estudio integrado en la zona de Iquitos, Perú. Annales Universitatis Turkuensis Ser. A II 114: 481-490. 